\title{
Generalized van der Waals equation for nonequilibrium fluids
}

\author{
D. Jou and C. Pérez-García \\ Departament de Termologia, Universitat Autónoma de Barcelona, \\ Bellaterra, Catalonia, Spain \\ (Received 18 April 1983)
}

\begin{abstract}
The inclusion of dissipative fluxes in the set of independent thermodynamic variables leads, among other features, to a modification of the equations of state. Here, we introduce into the van der Waals equation the generalized pressure obtained in extended irreversible thermodynamics and analyze the influence of a shear stress or a heat flux on the critical point of a fluid.
\end{abstract}

Some interest has been devoted to the formulation of a thermodynamic framework for nonequilibrium fluids including the dissipative fluxes (heat flux, viscous pressure tensor) as independent variables. ${ }^{1-6}$ In the usual descriptions, dissipative fluxes are considered as fast variables, and are therefore excluded from the set of basic independent variables, namely, the slow or conserved ones. However, if some experiment is done on the system at a frequency whose inverse may be comparable to the relaxation time of the fluxes, or if for some reason their relaxation times become long enough, the fluxes must be considered as "slow" independent variables in the thermodynamic formalism. The inclusion of the fluxes as independent variables of the fundamental equation of the system leads to a number of modifications of the usual thermodynamic framework, not only at the level of constitutive equations, ${ }^{2-4}$ but also in fluctuation theory ${ }^{5}$ and in the equations of state. ${ }^{1,6}$ This latter aspect has not been explored enough, and its consequences are mainly unknown. The purpose of this Brief Report is to show one of the possible applications of these nonequilibrium corrections which shows both the scope and the present limitations of the macroscopic theory. With this aim, we analyze here the influence of nonequilibrium parameters on the critical point of fluids, a problem which has interested some authors in the last years. ${ }^{7-12}$ In particular, we have studied the influence of shear stress and of heat flux on the critical point of a fluid. Though too simple in its present form to fit the experimental quantitative results, our model allows, however, for some nonclassical effects, not included in the usual theories of irreversible thermodynamics.

For a simple fluid under a shear stress, the theory of extended irreversible thermodynamics leads for the free energy $f$ per mole to the following differential relation ${ }^{1-3}$ :

$$
d f=-s d T-p d v+(\tau v / 2 \eta) \dot{\overrightarrow{\mathrm{P}}} v: d \dot{\overrightarrow{\mathrm{P}}} v,
$$

where $s$ and $v$ are, respectively, the entropy and volume per mole, $p$ and $T$ the pressure and absolute temperature, $\dot{\overrightarrow{\mathrm{P}}} v$ the traceless viscous pressure tensor, $\eta$ the shear viscosity, and $\tau$ the relaxation time of $\dot{\overrightarrow{\mathrm{P}}}^{v}$. For the sake of simplicity, and in accordance with the indications of experimental results in a range of densities, we assume that the relaxation time $\tau$ is linear in $v$, i.e., inversely proportional to the density of the fluid. Furthermore, we assume that the viscosity is independent of $v$, i.e., of density, though it depends on temperature. With these hypotheses, a simple explicit expression for the pressure $p$ may be obtained from the equality of second-order mixed derivatives of (1), namely,

$$
p=p_{0}-(\tau / 2 \eta) \dot{\overrightarrow{\mathrm{P}}} v: \dot{\overrightarrow{\mathrm{P}}} v
$$

Here, $p_{0}$ stands for the local-equilibrium pressure and the double dot indicates double contraction of the corresponding tensors.

Obviously, the thermodynamic theory cannot predict the explicit form of the equation of state, which must be obtained from statistical theory or from experimental work. As an explicit form of the equation of state, we take here the van der Waals equation, which is the simplest one leading to a critical point. Now, besides the usual correction $p=p_{\text {id }}-a v^{-2}$ to the pressure $p_{\text {id }}$ of the ideal gas, due to attractive interactions among molecules, we must consider also the nonequilibrium correction obtained in (2). In such a way we are led directly to the following generalized van der Waals equation,

$$
\left(p+a v^{-2}+\tau \eta \gamma^{2}\right)(v-b)=R T .
$$

Here, $R$ is the constant of gases, $a$ and $b$ are the usual parameters of the van der Waals equation, and we have considered a Couette plane flow with shear rate $\gamma$, so that $\dot{\mathrm{p}} v: \dot{\mathrm{P}}^{v}=2 \eta^{2} \gamma^{2}$.

When the conditions defining the critical point,

$$
\left(\frac{\partial p}{\partial v}\right)_{T, \gamma}=0 \text { and }\left(\frac{\partial^{2} p}{\partial v^{2}}\right)_{T, \gamma}=0 \text {, }
$$

are applied to Eq. (3), one obtains for the critical volume, pressure, and temperature, up to the first order in $\gamma^{2}$, the following values:

$$
\begin{aligned}
& v_{c}(\gamma)=v_{c}-\frac{8}{9}\left(v_{c}^{2} / R T_{c}\right) \tau \eta \gamma^{2}, \\
& p_{c}(\gamma)=p_{c}-\frac{17}{3} \tau \eta \gamma^{2}, \\
& T_{c}(\gamma)=T_{c}-\frac{4}{9}\left(v_{c} / R\right) \tau \eta \gamma^{2},
\end{aligned}
$$

where $v_{c}=3 b, \quad p_{c}=a / 27 b^{2}$, and $T_{c}=8 a / 27 R b$ are the respective critical values in equilibrium.

An analogous formulation may be developed for the case when the fluid is under a heat flux. In this case, the free energy per mole is, instead of (1),

$$
d f=-s d T-p d v+\left(\tau^{\prime} v / \lambda T\right) \overrightarrow{\mathrm{q}} \cdot d \overrightarrow{\mathrm{q}}
$$

with $\vec{q}$ the heat flux, $\lambda$ the thermal conductivity, and $\tau^{\prime}$ the relaxation time of $\overrightarrow{\mathrm{q}}$. Assuming, as in the previous case, that $\tau^{\prime}$ is linearly proportional to $v$ and that $\lambda$ is independent 
of $v$, one gets for the generalized pressure

$$
p=p_{0}-\left(\tau^{\prime} / \lambda T\right) \overrightarrow{\mathrm{q}}^{2} \text {. }
$$

This leads to a generalized van der Waals equation of the form

$$
\left[p+a v^{-2}+\tau^{\prime}(\lambda T)^{-1} \overrightarrow{\mathrm{q}}^{2}\right](v-b)=R T
$$

whose corresponding critical values of volume, density, and temperature are

$$
\begin{aligned}
& v_{c}(q)=v_{c}-\frac{8}{9}\left(v_{c}^{2} / R T_{c}\right)\left(\tau^{\prime} / \lambda T_{c}\right) \overrightarrow{\mathrm{q}}^{2}, \\
& p_{c}(q)=p_{c}-\frac{17}{3}\left(\tau^{\prime} / \lambda T_{c}\right) \overrightarrow{\mathrm{q}}^{2}, \\
& T_{c}(q)=T_{c}-\frac{4}{9}\left(v_{c} / R\right)\left(\tau^{\prime} / \lambda T_{c}\right) \overrightarrow{\mathrm{q}}^{2} .
\end{aligned}
$$

Our results may be partially compared with those obtained by Onuki and Kawasaki in a series of papers. ${ }^{9-11}$ In the case when the fluid is under a shear stress and using renormalization-group techniques, they obtain, for the critical value of the temperature,

$$
T_{c}(\gamma)=T_{c}-0.0832 \epsilon\left(k_{c} \xi_{0}\right)^{1 / \nu} .
$$

Here, $\epsilon=4-d, d$ being the dimensionality of the system; $\xi_{0}$ is a length related to the correlation length $\xi$ of the system by $\xi=\xi_{0}\left[\left(T / T_{c}\right)-1\right]^{-\nu}$, with $\nu$ the critical exponent which describes the divergence of $\xi$ when $T$ approaches the critical temperature. The critical wave number $k_{c}$ is determined by equating the decay rate $\Gamma_{k}$ of the order parameter to the shear rate $\gamma$. The decay rate is given in the dynamic scaling theory by $\Gamma_{k}=k^{2} \Omega(k \xi)$, where $z$ is a critical exponent. In the problem of Onuki and Kawasaki, and for $k \xi>1, z=3$, and $\Omega(k \xi)=k_{B} T / 16 \eta$, so that their result may be written as

$$
T_{c}(\gamma)=T_{c}-0.0832 T_{c}\left(16 \eta \xi_{0}^{3} / k_{B} T_{c}\right)^{1 / z \nu} \gamma^{1 / z \nu} .
$$

For an aniline-cyclohexane mixture in three dimensions $(z=3, v \approx 0.63)$, (11) takes the form

$$
T_{c}(\gamma)=T_{c}-1.3 \times 10^{-4} \gamma^{0.53} \mathrm{~K} .
$$

This is in good agreement with the experimental results of Beysens and Gbamadassi. ${ }^{12}$

In order to facilitate the comparison of our result (5) for the nonequilibrium critical temperature, we may consider in the corresponding expression in (5) that $\tau \approx \eta / p_{c}$, so that we obtain

$$
T_{c}(\gamma)=T_{c}-6^{-3} T_{c}\left(16 \eta v_{c} / R T_{c}\right)^{2} \gamma^{2} .
$$

The comparison between (11) and (13) is more direct if we define a length $l$ as $l=\left(v_{c} / N_{A}\right)^{1 / 3}$, where $N_{A}$ is Avogadro's number. We note, however, that the exponents and the coefficients of (11) and (13), respectively, do not coincide. Of course, this is not surprising, since our theory, as well as the classical van der Waals equation, behaves as a kind of "mean-field" phenomenological theory with an analytic development. In the case of the van der Waals equation, the mean field is related to the interactions among molecules, while in our case, the mean field is related to the nonequilibrium distortion of the pair-correlation function, which is the physical origin of the corrections to the pressure. This nonequilibrium correction seems to be a nonana- lytic function of the shear rate, according to molecular dynamics computations, ${ }^{7,8}$ this being the cause of the disagreement with our macroscopic analytic theory. A more general phenomenological analysis would be possible along the lines of extended irreversible thermodynamics ${ }^{6}$ but, lacking more precise data, it would be merely formal.

Let us note also that in the case of helium near the superfluid transition, Onuki ${ }^{11}$ has obtained, for the surface critical temperature, the following relation:

$$
T_{\mathrm{sc}}(q)=T_{\mathrm{sc}}-11.7 \times 10^{-5} q^{3 / 4} \mathrm{~K},
$$

where $T_{\mathrm{sc}}$ is now $T_{\lambda}$, the $\lambda$ temperature. This is in fair agreement with the temperature at the interface of $\mathrm{He} I$ and He II under heat flow. ${ }^{13,14}$ Also in this case, the exponent of the correction does not coincide with our exponent, due to the usual nonanalytic behavior of the equations of state near the critical point.

On the other hand, Hanley and Evans ${ }^{7,8}$ have carried out a molecular dynamics computation of nonequilibrium corrections to the pressure, and have obtained $p=p_{0}+p_{1} \gamma^{3 / 2}$, in the case of a fluid under shear. In spite of its nonanalytical behavior, this development does not fit the exponent predicted by the microscopic theory of Onuki and Kawasaki.

An evaluation of the coefficient $\eta^{2} v_{c}^{2} / R^{2} T_{c}$ appearing in (5) for noble gases $\left(v_{c} \approx 10^{-5} \mathrm{~m}^{3} / \mathrm{mol}, T_{c} \approx 10^{2} \mathrm{~K}\right.$, $\eta \cong 10^{-5} \mathrm{~N} \mathrm{~s} / \mathrm{m}^{2}$ ) leads to a value $\eta^{2} v_{c}^{2} / R^{2} T_{c} \approx 10^{-24} \mathrm{~s}^{2}$, so that in order for the corrections in (5) to be appreciable, one should use perturbations with a frequency of the order $10^{12} \mathrm{~Hz}$. This result is quite logical, since the relaxation times of the fluxes in simple fluids are of the order of $10^{-12}$ s. However, in viscoelastic fluids these relaxation times are usually much longer, so that these effects may be more easily appreciated. Furthermore, near a critical point it is possible that collective effects may contribute to making the relaxation times considerably longer than in the usual situation, but this is evidently far beyond the scope of the present simple formalism.

As a conclusion of this Brief Report we outline that the nonequilibrium corrections to the equations of state predicted by extended irreversible thermodynamics are not merely academic, but that they may have an experimental meaning. The dynamical equations may indeed influence the equations of state, as is shown by the presence of the dynamical critical exponent $z$ in the nonequilibrium corrections to the critical point in (11). Though in its present form our formalism is too simple to predict the correct values of the exponents and the coefficients-a usual shortcoming of phenomenological mean-field theories-it may provide a starting point for a more general development. Such a framework, which includes in the equations of state not only equilibrium but also nonequilibrium dynamical properties, may provide a natural and direct treatment to some phenomena such as the one dealt within this paper.

We thank Professor J. Casas-Vázquez for his valuable suggestions. This work has been partially supported by a grant from the Comisión Asesora de Investigación Científica y Técnica of the Spanish Government and by the North Atlantic Treaty Organization (NATO) Research Grant No. 0355/83. 
${ }^{1}$ R. E. Nettleton, Phys. Fluids 2,256 (1959); 3, 213 (1960).

${ }^{2}$ I. Müller, Z. Phys. 198, 329 (1967).

${ }^{3}$ D. Jou, J. Casas-Vázquez, and G. Lebon, J. Non-Equilib. Thermodynam. 4, 349 (1979); G. Lebon, D. Jou, and J. Casas-Vázquez, J. Phys. A 13,275 (1980).

${ }^{4}$ J. A. Robles-Domínguez, B. Silva, and L. S. García-Colín, Physica (Utrecht) 106A, 539 (1981); B. C. Eu, J. Chem. Phys. 73, 2958 (1980); F. Bampi and A. Morro, J. Math. Phys. 21, 1201 (1980).

${ }^{5}$ D. Jou, J. E. Llebot, and J. Casas-Vázquez, Phys. Rev. A $\underline{25}, 508$ (1982); 25, 3277 (1982).

${ }^{6}$ C. Pérez-García and D. Jou, J. Non-Equilib. Thermodynam. Z, 191 (1982).
${ }^{7}$ D. J. Evans and H. J. M. Hanley, Phys. Lett. 80A, 175 (1980).

${ }^{8}$ H. J. M. Hanley and D. J. Evans, J. Chem. Phys. 76, 3225 (1982).

${ }^{9}$ A. Onuki, K. Yamazaki, and K. Kawasaki, Ann. Phys. (N.Y.) 131 , 217 (1981).

${ }^{10}$ A. Onuki and K. Kawasaki, Ann. Phys. (N.Y.) $\underline{121}, 456$ (1979); Prog. Theor. Phys. 63, 122 (1980).

${ }^{11}$ A. Onuki, Prog. Theor. Phys. 63, 1854 (1980); 64, 1902 (1980).

${ }^{12}$ D. Beysens and M. Gbamadassi, J. Phys. (Paris) Lett. 40, L565 (1979); Phys. Rev. Lett. 43, 1253 (1979).

${ }^{13}$ K. D. Erben and E. Pobell, Phys. Lett. 26A, 368 (1968).

${ }^{14}$ S. M. Bhagat and R. A. Lasken, Phys. Rev. A $\underline{3}, 577$ (1980); $\underline{5}$, 2297 (1972). 